\title{
ATRIBUTOS FÍSICOS E QUÍMICOS DE UM ARGISSOLO VERMELHO, EM POMAR ORGÂNICO DE CITROS COM MANEJO DA VEGETAÇÃO NAS ENTRELINHAS ${ }^{1}$
}

\author{
JÔNATAN MÜLLER ${ }^{2}$, RENATO LEVIEN ${ }^{3}$, CARLOS RICARDO TREIN ${ }^{3}$, \\ LETÍCIA SEQUINATTO ${ }^{4}$, MICHAEL MAZURANA ${ }^{2}$, \\ SIDINEI LEANDRO KLÖCKNER STÜRMER ${ }^{5}$, ROGÉRIO PIETRZACKA ${ }^{6}$
}

RESUMO-O manejo em pomares orgânicos de citros é diferenciado em relação aos pomares convencionais. Deste modo, objetivou-se avaliar alguns atributos físicos e químicos de um Argissolo espessarênico e produtividade do pomar de tangerineiras, cv. montenegrina, sob sistema orgânico de produção, com diferentes manejos da vegetação nas entrelinhas. Os tratamentos estudados foram: gradagem, roçada, acamamento com rolo-faca e com arraste de tronco. A avaliação dos atributos físicos ocorreu sob a projeção da copa e na área de tráfego de máquinas, nas camadas de 0,0-0,1 e 0,1-0,2 m. A fertilidade química do solo foi determinada nas profundidades de $0,0-0,1 ; 0,1-0,2$ e $0,2-0,4 \mathrm{~m}$, enquanto a produtividade de frutos foi estimada a partir das plantas centrais de cada parcela. $\mathrm{O}$ tráfego de máquinas influenciou negativamente nos atributos físicos do solo abaixo da interface pneu/solo, embora não tenha sido restritivo à produtividade de frutos. Em todos os tratamentos, houve incremento de matéria orgânica na camada superficial do solo e dos teores de $\mathrm{P}, \mathrm{K}^{+}$, $\mathrm{Ca}^{2+}$ e $\mathrm{Mg}^{2+}$ nas três camadas de solo em relação à área adjacente com vegetação nativa. $\mathrm{O}$ manejo com gradagem apresentou produtividade significativamente maior em relação ao manejo com roçada.

Termos para indexação: agricultura orgânica, física do solo, citricultura.

\section{PHYSICAL AND CHEMICAL ATTRIBUTES OF A TYPIC ULTISOL IN ORGANIC CITRUS ORCHARD WITH WEED CONTROL BETWEEN THE LINES}

\begin{abstract}
The management in organic citrus orchards is differentiated with respect to conventional orchards. Thus, it was aimed to evaluate some physical and chemical attributes of a typic ultisol and productivity of a mandarin orchard, cv. Montenegrin under organic production system, with different vegetation management between the lines. The treatments were: harrowing, mowing, lodging with coulter and log dragging. The evaluation of the physical attributes occurred under the canopy and in the machinery traffic area, in the depths of $0.0-0.1$ and $0.1-0.2 \mathrm{~m}$. The chemical fertility of the soil was determined in the layer of 0.0-0.1, 0.1-0.2 and 0.2-0.4 m, while the fruit yield was estimated from central plants of each portion. Machine traffic negatively affected the physical attributes of soil below the interface patch, although it was not restrictive to fruit yield. In all treatments there was an increase of organic matter in the topsoil, and the levels of $\mathrm{P}, \mathrm{K}^{+}, \mathrm{Ca}^{2+}$ and $\mathrm{Mg}^{2+}$ in the three soil layers in relation to the adjacent area with native vegetation. The handling with harrowing showed significantly higher productivity in relation to the management with mowing.
\end{abstract}

Index terms: organic farming, soil physics, citrus.

\footnotetext{
1(001-11). Recebido em: 03-01-2011. Aceito para publicação em: 06-09-2011.

${ }^{2} \mathrm{Eng}^{\mathrm{o}}$. Agr $^{\mathrm{o}}$, Mestrando em Ciência do Solo, Departamento de Solos, Faculdade de Agronomia/UFRGS, Porto Alegre-RS, CEP $91540-$ 000,CNPq, E-mails: jonatanmuller@hotmail.com; michael.mazurana@gmail.com.

${ }^{3}$ Prof. Titular, Departamento de Solos, Faculdade de Agronomia/UFRGS, Av. Bento Gonçalves, 7712, CEP 91540-000, Porto AlegreRS E-mails: renatole@gmail.com; trein@ufrgs.br.

${ }^{4}$ Pós-doutoranda em Ciência do Solo, Departamento de Solos, Centro de Ciências Rurais/UFSM, Av. Roraima 1000, Santa Maria-RS, CEP 97105-900, CNPq, E-mails: letisequinatto@gmail.com.

${ }_{5}^{5}$ Doutorando em Ciência do Solo, Departamento de Solos, Faculdade de Agronomia/UFRGS, Porto Alegre-RS, CEP 91540-000, CNPq, E-mail: sidineileandro@gmail.com.

${ }^{6}$ Mestre em Ciência do Solo, Departamento de Solos, Faculdade de Agronomia/UFRGS, Porto Alegre-RS, CEP 91540-000, E-mail: rogerioagro@yahoo.com.br.
} 


\section{INTRODUÇÃO}

A citricultura ocupa lugar de destaque no Brasil, sendo de grande importância econômica e social. No Rio Grande do Sul, as frutas cítricas apresentam área cultivada de 42.000 ha, concentrando-se principalmente em pequenas propriedades familiares (BONINE; JOÃO, 2002), obtendo produção, em 2007, de mais de 540 mil toneladas de citros, com produtividade média de 16,4 t/ha (IBGE, 2009).

Segundo Homma (2005), um número crescente de pesquisadores aponta para a necessidade de buscar sistemas alternativos de produção para a citricultura, em vista da ampliação do mercado de produtos orgânicos certificados.

No sistema orgânico de produção, muitos agricultores optam pela manutenção permanente da cobertura vegetal nas entrelinhas do pomar, manejando seu crescimento com roçadas ou tombamento da vegetação, além de realizarem adubações com adição de compostos orgânicos e biofertilizantes. Entretanto, a manutenção de cobertura vegetal nas entrelinhas dos pomares condiciona a competição por nutrientes e água, podendo comprometer, de forma significativa, a produtividade das plantas citrícolas, principalmente em solos arenosos que apresentam baixa retenção de água (NEVES; DECHEN, 2001).

Limitações impostas ao desenvolvimento do sistema radicular das plantas ocorrem pela compactação do solo, em função do tráfego excessivo de máquinas em operações de adubação, pulverização e manejo das plantas de cobertura na entrelinha (TERSI; ROSA, 1995), representando uma das causas da redução de produtividade dos pomares.

A compactação ocorre principalmente nas entrelinhas de plantio, em razão do tráfego de máquinas e implementos ocorrer sempre no mesmo local. Esse tráfego contínuo faz com que seja transferida uma determinada força por unidade de área, em virtude da interface pneu/solo, gerando alterações físicas negativas para o desenvolvimento do sistema radicular dos citros (MINATEL et al., 2006). Fidalski et al. (2007) apontam que o manejo mecanizado na cultura dos citros resulta na heterogeneidade das propriedades físicas do solo. A pesquisa, até o momento, mostra resultados conflitantes quanto ao manejo de vegetação nas entrelinhas do pomar e seus reflexos na produtividade dos citros, apresentando tanto interações positivas (AULER et al., 2008) como negativas (WRIGHT et al., 2003).

Deste modo, o objetivo do trabalho foi avaliar atributos físicos e químicos de um Argissolo espessarênico e a produtividade do pomar orgânico de citros, conduzidos com diferentes formas de manejo da vegetação espontânea nas entrelinhas do pomar de tangerineiras.

\section{MATERIAL E MÉTODOS}

O experimento foi conduzido em pomar orgânico de tangerineiras, localizado na Região Fisiográfica da Depressão Central do Rio Grande do Sul. O solo é classificado como Argissolo Vermelho distrófico espessarênico. O clima da região é Cfa subtropical úmido, com temperatura média anual de $19,4^{\circ} \mathrm{C}$ e precipitação anual média de $1.365 \mathrm{~mm}$, com períodos de escassez de chuva no verão.

O talhão do experimento foi implantado em 1990 por manejo convencional, com plantio das mudas de tangerineiras (Citrus deliciosa), cv. Montenegrina, com espaçamento de $3 \mathrm{~m}$ entre plantas e 6,5 m entre linhas. Em 1998, a área do pomar foi convertida para o sistema orgânico de cultivo. As adubações são realizadas em intervalos bianuais, com a aplicação de $30 \mathrm{~m}^{3}$ ha-1 de composto orgânico sólido preparado pela cooperativa Ecocitrus e, em intervalos anuais, $30 \mathrm{~m}^{3} \mathrm{ha}^{-1}$ de biofertilizante. Os implementos utilizados para realizar os tratos culturais são tracionados por um trator $4 \times 2$ com $55 \mathrm{CV}$ de potência no motor e peso total lastrado de $2.430 \mathrm{~kg}$. Em média, são realizadas 10 passagens de máquinas pelas entrelinhas do talhão durante o ano agrícola.

A área útil de cada parcela do experimento é composta por três linhas de tangerineiras com duas entrelinhas por tratamento, constituídas por 15 tangerineiras (5 plantas por linha) e vegetação espontânea. Os tratamentos utilizados foram: T1- solo gradeado com grade niveladora em tandem, visando à incorporação parcial da vegetação no solo em profundidade aproximada de 0,1 m; T2 - acamamento com arraste de dois segmentos de tronco de madeira dispostos em 'V' com dispositivos para acoplamento no sistema de três pontos do trator; T3 - corte mecânico com roçadeira, e T4 - acamamento e corte utilizando rolo-faca com massa de $600 \mathrm{~kg}$.

Para a avaliação dos atributos físicos, o delineamento experimental foi em blocos ao acaso, com parcelas subsubdivididas e quatro repetições, considerando-se os tratamentos principais (T1, T2, T3 e T4), os locais de tráfego dos rodados e a projeção da copa (T e PC) como subtratamentos, e as profundidades $(0,0-0,1$ e $0,1-0,2 \mathrm{~m})$ como subsubtratamentos. Nos atributos de fertilidade do solo e de produtividade do pomar, o delineamento utilizado foi o inteiramente casualizado.

As amostras de solo para análise química foram coletadas em três camadas do solo $(0,0-0,1$; $0,1-0,2$ e 0,2-0,4 m). Para fins de comparação, tam- 
bém foram coletadas amostras de solo, nas três profundidades de estudo, em área de vegetação nativa, adjacente ao talhão do experimento.

A determinação da resistência mecânica do solo à penetração (RP) foi efetuada, utilizando-se de Penetrômetro Digital dotado de sistema eletrônico de medição e aquisição dos dados de força aplicada a cada profundidade no solo, na projeção da copa (PC), entre rodados (ER) e no tráfego de rodados $(\mathrm{T})$, até a profundidade de $0,4 \mathrm{~m}$. Juntamente com os dados de $\mathrm{RP}$, quantificou-se a umidade gravimétrica do solo.

A coleta de amostras de solo para a determinação da densidade do solo, macroporosidade, microporosidade e porosidade total ocorreu 45 dias após o manejo das plantas espontâneas, em outubro. Coletaram-se amostras de solo, em duas camadas (0,0-0,1 e 0,1-0,2 m) e em dois locais (T e PC).

Para a determinação da produtividade nos diferentes tratamentos principais, foram contados e pesados os frutos das três plantas centrais dentro de cada parcela. A estimativa de produção foi calculada para 500 plantas/ha devido ao seu espaçamento $(6,5$ $\mathrm{m} \times 3 \mathrm{~m})$.

As variáveis estudadas foram submetidas à análise de variância, em nível de $(\mathrm{p}<0,05)$ de significância entre médias, pelo teste de Tukey, com uso do programa estatístico SPSS.

\section{RESULTADOS E DISCUSSÃO}

Os valores de $\mathrm{pH}$ do solo em todos os tratamentos foram altos até os $0,4 \mathrm{~m}$ (Tabela 1). Esses valores decresceram ao longo do perfil do solo em vista de a adubação orgânica ser realizada em superfície, corroborando os estudos de Panzenhagen et al. (2008), que observaram que o pH dos solos de pomares com aplicação de composto orgânico e biofertilizante líquido é invariavelmente alto (acima de 6,0), devido a sua composição apresentar característica de alcalinidade .

A constante adição de compostos orgânicos, bem como a permanência da vegetação espontânea na superfície do solo, gerou aumento nos teores de matéria orgânica e de nutrientes na camada de 0,0 - 0,1 $\mathrm{m}$, se comparados às demais profundidades que, por sua vez, tiveram valores semelhantes. Pode-se inferir que os valores de MO diagnosticados na camada de 0,0-0,1 m são responsáveis, em grande parte, pelos valores da CTC e disponibilidade de $\mathrm{P}, \mathrm{K}^{+}, \mathrm{Ca}^{2+}, \mathrm{Mg}^{2+}$, visto que o teor de argila nesta camada é de $0,1 \mathrm{~kg}$ $\mathrm{kg}^{-1}$ de solo. Estes resultados corroboram o trabalho de Silva et al. (2004), que apontam a contribuição da MO variando de 20 a $70 \%$ da CTC, na maioria dos solos, enquanto Fávero et al. (2000) demonstraram a influência de plantas espontâneas na ciclagem de $\mathrm{P}, \mathrm{K}^{+}$e $\mathrm{Mg}^{2+}$, chegando a acumular até três vezes mais nutrientes quando comparado com leguminosas tradicionalmente utilizadas como adubos verdes.

De acordo com Magalhães (2004), o pH mais favorável ao desenvolvimento de plantas citrícolas situa-se entre 6,0 e 7,0, em cuja faixa é maior a disponibilidade dos nutrientes catiônicos. Como 70 a $80 \%$ das raízes ativas se encontram a menos de 0,5 $\mathrm{m}$ de profundidade (CRUZ et al., 2005; NOOLING, 1992), a fertilidade do solo apresentada na Tabela 1 não é fator responsável pela diferenciação entre a produtividade de frutos dos tratamentos principais, porém pode limitá-la. Segundo Malavolta (1994), valores crescentes de $\mathrm{pH}$ geram uma diminuição linear da disponibilidade de micronutrientes catiônicos, essenciais para o metabolismo das plantas.

Os ensaios de RP apresentaram certa similaridade nos tratamentos $\mathrm{T} 1, \mathrm{~T} 2, \mathrm{~T} 3$ e $\mathrm{T} 4$, como entre os subtratamentos PC e ER, com exceção do T, que abaixo de $0,15 \mathrm{~m}$ apresentou valores altos . Até 0,1 $\mathrm{m}$ de profundidade, o solo exibiu baixos valores de RP em todos os tratamentos e subtratamentos, mesmo nos locais sob influência direta do tráfego de rodados. Esses resultados estão relacionados ao teor de matéria orgânica (MO) que se apresenta com teores médios na camada superficial (Tabela 1). Six et al. (2002) afirmam que, através da estabilização bioquímica, a MO pode diminuir o potencial de compactação do solo.

Na região do tráfego de rodados, apresentada na Figura 1, os valores de RP são considerados críticos, pois Silva et al. (2004) mencionam que valores de resistência mecânica à penetração acima de 2.000 $\mathrm{kPa}$ limitam o crescimento da planta.

Já na região ER, visualiza-se que a compactação gerada no T influencia a RP deste local, sendo identificadas as faixas de maior RP na profundidade de 0,15 a $0,25 \mathrm{~m}$. Isso pode ocorrer porque, apesar de as tensões se propagarem quase que em sua totalidade de forma vertical no solo, parte dessas tensões também se propaga lateralmente (TREIN, 1995). De modo geral, os resultados de RP na Figura 1apontam zonas heterogêneas de compactação nos subtratamentos, corroborando os resultados obtidos pelos autores (FIDALSKI et al., 2007).

A diferença de densidade do solo (Tabela 2) entre a projeção da copa e o rodado (com valores de Ds maiores na região do rodado em ambas as profundidades) sugere efeito residual do tráfego de máquinas.

As variações de micro e macroporosidade (Tabela 2) decorrem do local (PC e T) e da profundidade de coleta dos anéis, uma vez que a vegetação espontânea permanece na entrelinha do pomar 
conduzido de forma orgânica, e grande parcela da massa radicular da vegetação espontânea e das tangerineiras situa-se nas profundidades iniciais do solo, fato que pode acarretar a obtenção de anéis amostrais com grande quantidade de raízes, que, em laboratório, acabam influenciando nas determinações físicas do solo.

Os valores da porosidade total (PT) apresentaram variações na proporção entre macro e microporosidade, com maiores médias na camada de $0,0-0,1 \mathrm{~m}$, em ambos os locais (rodado e projeção da copa), em relação à segunda profundidade analisada. Demattê e Vitti (1992) relatam que os solos para cultivo com citros devem possuir valores de PT entre 0,4 e $0,55 \mathrm{~m}^{3} \mathrm{~m}^{-3}$, situação observada neste experimento em todos os tratamentos, exceto em $\mathrm{T} 4$, no $\mathrm{T}$, até $0,20 \mathrm{~m}$. Isso pode ter ocorrido devido à massa seca de plantas espontâneas (Tabela 4) em T4 ser, significativamente inferior em relação aos outros tratamentos de manejo das plantas de cobertura. Soane (1990) afirma que o acúmulo de resíduos vegetais na superfície do solo pode atenuar as cargas aplicadas pelas máquinas agrícolas, devido à sua elasticidade e ao aumento da área de contato efetiva entre o solo e os rodados. Desse modo, o tráfego em $\mathrm{T} 4$, provavelmente, gerou a diminuição da macroporosidade, obtendo-se, consequentemente, menor porosidade total em relação aos outros tratamentos.

Apesar de o tráfego de máquinas causar diminuição da PT do solo e aumentar a densidade na profundidade de $0,1-0,2 \mathrm{~m}$, ele não chega a ser um problema do ponto de vista do espaço poroso. Nesse particular, cita-se a influência do tipo de solo, menos propício à compactação por ter textura arenosa, bem como dos teores de MO presente na camada superficial do solo (Tabela 1), que minimiza o efeito da passagem do rodado no solo.

Avilan et al. (1980) afirmam que, em solos profundos, neste caso, Argissolo espessarênico, situados em regiões sujeitas a escassez de chuva, as raízes aprofundam-se mais e geralmente se mantêm nos limites da projeção da copa. Ao contrário, em solos rasos e com boa distribuição de chuvas, as raízes desenvolvem-se próximas à superfície e estendem-se 1 a 2 metros além da periferia da copa. Desse modo, as mudanças nas características físicas do solo, no local de tráfego das máquinas, não aparentam ser fator limitante ao desenvolvimento e produtividade das plantas citrícolas.

Nos dados de produtividade de frutos de tangerineiras (Tabela 3), o tratamento $\mathrm{T} 1$ apresenta as maiores médias entre os tratamentos para as variáveis analisadas, com exceção do peso médio de frutos, sendo as menores médias no tratamento T3, porém com maior peso médio de frutos. Quanto ao peso de frutos, houve diferença significativa entre os tratamentos T1 e T3. Apesar de o tratamento T1 apresentar maior número e peso de frutos, quando comparado o peso médio de frutos, nota-se que o tratamento T3 foi beneficiado, uma vez que, com menos frutos por planta, o peso por fruto foi maior. Este fato deve ser ressaltado devido à comercialização in natura do produto, tendo o consumidor preferência por frutos maiores.

Para a variável número de frutos, entre os tratamentos analisados, o tratamento $\mathrm{T} 1$ apresentou as maiores médias e diferiu significativamente do tratamento T2 e T3, sendo que, na produtividade estimada, esta diferença ocorreu apenas no tratamento T3. A diferenciação da produtividade entre estes tratamentos pode estar associada ao controle da vegetação espontânea em solo arenoso, com consequente redução da competição por água pelo sistema radicular e à taxa de evapotranspiração das espécies nas entrelinhas do pomar (PERIN et al., 2000), em região com déficit hídrico no período de desenvolvimento dos frutos.

Os valores de peso de frutos/planta apresentaram-se superiores ao valor médio de $25 \mathrm{~kg} /$ planta obtido neste talhão do pomar, em anos anteriores. A produtividade do pomar está de acordo com a média geral de 16,4 t ha ${ }^{-1}$, obtida no Estado do RS (IBGE, 2007).

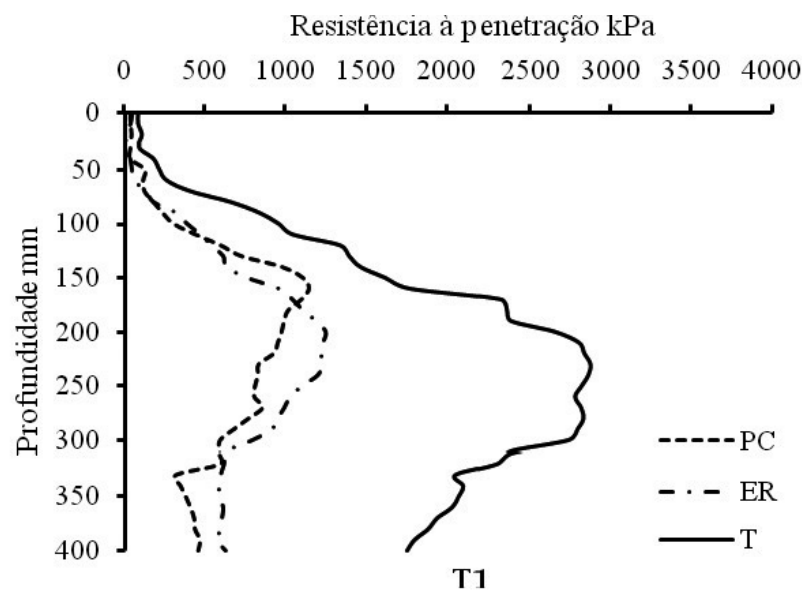

continua... 
continuação
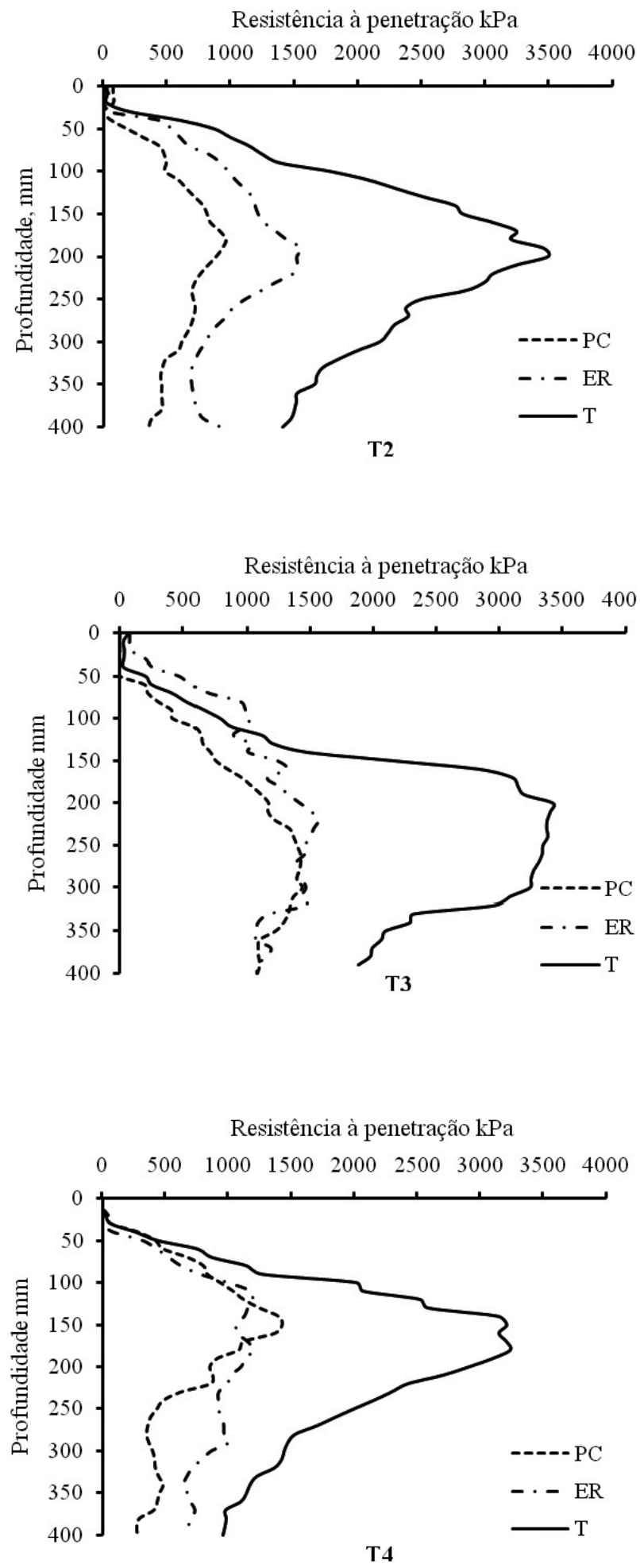

FIGURA 1 - Resistência mecânica do solo à penetração na projeção da copa (PC), entre rodados (ER) e tráfego de rodados $(\mathrm{T})$, nos diferentes tratamentos principais (T1, T2, T3 e T4), até $0,4 \mathrm{~m}$ de profundidade. A umidade gravimétrica do solo no momento da aquisição dos dados foi de $0,09 \mathrm{~kg} \mathrm{~kg}^{-1}$. 
TABELA 1 - Valores de pH, matéria orgânica, fósforo, potássio, capacidade de troca catiônica, cálcio e magnésio do solo, em três camadas coletadas do pomar de citrus, nos tratamentos principais.

\begin{tabular}{ccccccccc}
\hline \multirow{2}{*}{ Locais } & Prof. & $\mathbf{p H}$ & $\mathbf{M O}$ & $\mathbf{P}$ & $\mathbf{K}$ & $\mathbf{C T C}$ & $\mathbf{C a}$ & $\mathbf{M g}$ \\
\cline { 2 - 9 } & $---\mathbf{m}---$ & & $\mathbf{~}$ & $----\mathbf{m g} \mathbf{d m}^{-3}----$ & $-------\mathbf{c m o l}_{\mathbf{c}} \mathbf{d m}^{-3}--------$ \\
\hline \multirow{2}{*}{ T 1 } & $0,0-0,1$ & $7,6 \mathrm{~A}$ & $5,0 \mathrm{M}$ & $145 \mathrm{MA}$ & $80 \mathrm{~A}$ & $14,5 \mathrm{M}$ & $12,4 \mathrm{~A}$ & $1,1 \mathrm{~A}$ \\
& $0,1-0,2$ & $7,5 \mathrm{~A}$ & $1,3 \mathrm{~B}$ & $31 \mathrm{~A}$ & $37 \mathrm{~B}$ & $6,0 \mathrm{M}$ & $4,6 \mathrm{~A}$ & $0,4 \mathrm{~B}$ \\
& $0,2-0,4$ & $6,9 \mathrm{~A}$ & $0,9 \mathrm{~B}$ & $15 \mathrm{M}$ & $43 \mathrm{M}$ & $6,2 \mathrm{M}$ & $4,2 \mathrm{~A}$ & $0,4 \mathrm{~B}$ \\
\hline \multirow{2}{*}{ T 2 } & $0,0-0,1$ & $7,6 \mathrm{~A}$ & $3,7 \mathrm{M}$ & $143 \mathrm{MA}$ & $83 \mathrm{~A}$ & $12,5 \mathrm{M}$ & $10,7 \mathrm{~A}$ & $0,8 \mathrm{M}$ \\
& $0,1-0,2$ & $7,5 \mathrm{~A}$ & $1,2 \mathrm{~B}$ & $27 \mathrm{~A}$ & $42 \mathrm{M}$ & $6,1 \mathrm{M}$ & $4,8 \mathrm{~A}$ & $0,4 \mathrm{~B}$ \\
& $0,2-0,4$ & $6,9 \mathrm{~A}$ & $1,0 \mathrm{~B}$ & $15 \mathrm{M}$ & $32 \mathrm{M}$ & $6,1 \mathrm{M}$ & $4,0 \mathrm{M}$ & $0,4 \mathrm{~B}$ \\
\hline \multirow{2}{*}{ T 3 } & $0,0-0,1$ & $7,7 \mathrm{~A}$ & $3,1 \mathrm{M}$ & $122 \mathrm{MA}$ & $94 \mathrm{~A}$ & $10,7 \mathrm{M}$ & $8,9 \mathrm{~A}$ & $0,8 \mathrm{M}$ \\
& $0,1-0,2$ & $7,3 \mathrm{~A}$ & $1,1 \mathrm{~B}$ & $22 \mathrm{~A}$ & $47 \mathrm{M}$ & $5,6 \mathrm{M}$ & $4,1 \mathrm{~A}$ & $0,4 \mathrm{~B}$ \\
& $0,2-0,4$ & $6,7 \mathrm{~A}$ & $1,1 \mathrm{~B}$ & $18 \mathrm{M}$ & $30 \mathrm{~B}$ & $6,2 \mathrm{M}$ & $4,0 \mathrm{M}$ & $0,4 \mathrm{~B}$ \\
\hline \multirow{2}{*}{ T 4 } & $0,0-0,1$ & $7,5 \mathrm{~A}$ & $3,9 \mathrm{M}$ & $125 \mathrm{MA}$ & $116 \mathrm{~A}$ & $11,5 \mathrm{M}$ & $9,2 \mathrm{~A}$ & $1,1 \mathrm{~A}$ \\
& $0,1-0,2$ & $7,0 \mathrm{~A}$ & $1,4 \mathrm{~B}$ & $25 \mathrm{~A}$ & $62 \mathrm{~A}$ & $6,2 \mathrm{M}$ & $4,2 \mathrm{~A}$ & $0,6 \mathrm{M}$ \\
& $0,2-0,4$ & $6,2 \mathrm{~A}$ & $1,1 \mathrm{~B}$ & $16 \mathrm{M}$ & $42 \mathrm{M}$ & $6,8 \mathrm{M}$ & $3,5 \mathrm{M}$ & $0,7 \mathrm{M}$ \\
\hline \multirow{2}{*}{ Mata } & $0,0-0,1$ & $5,2 \mathrm{~B}$ & $1,8 \mathrm{~B}$ & $4 \mathrm{MB}$ & $42 \mathrm{~B}$ & $7,5 \mathrm{M}$ & $1,9 \mathrm{~B}$ & $1,1 \mathrm{~A}$ \\
& $0,1-0,2$ & $4,7 \mathrm{MB}$ & $1,1 \mathrm{~B}$ & $2 \mathrm{MB}$ & $22 \mathrm{MB}$ & $6,0 \mathrm{M}$ & $1,2 \mathrm{~B}$ & $0,9 \mathrm{M}$ \\
& $0,2-0,4$ & $4,5 \mathrm{MB}$ & $1,0 \mathrm{~B}$ & $2 \mathrm{MB}$ & $25 \mathrm{MB}$ & $6,6 \mathrm{M}$ & $1,0 \mathrm{~B}$ & $0,7 \mathrm{M}$ \\
\hline
\end{tabular}

Classificação dos valores em faixas segundo COMISSÃO (2004). MA=muito alto; $\mathrm{A}=$ alto; $\mathrm{M}=$ médio; $\mathrm{B}=$ baixo; $\mathrm{MB}=\mathrm{M} u i t o$ baixo.

TABELA 2 - Densidade, macro, micro e porosidade total do solo avaliada na região do rodado e projeção da copa, em duas profundidades, para os diferentes manejos da vegetação no pomar de citurs.

\begin{tabular}{|c|c|c|c|c|c|c|c|c|c|}
\hline \multirow{3}{*}{ Atributo } & \multirow{3}{*}{ Tratamento } & \multicolumn{4}{|c|}{ Rodado } & \multicolumn{4}{|c|}{ Projeção da Copa } \\
\hline & & $0,0-0,1$ & & 0,1 & & $\mathbf{0 , 0}$ & & & 0,2 \\
\hline & & \multicolumn{4}{|c|}{ - } & \multicolumn{4}{|c|}{ - } \\
\hline \multirow{4}{*}{$\begin{array}{c}\text { Densidade } \\
\text { do solo } \\
\left(\mathrm{Mg} \mathrm{m}^{-3}\right)\end{array}$} & Grade & 1,29 & $A b c$ & 1,63 & $\mathrm{Aa}$ & 1,24 & Ac & 1,51 & $\mathrm{Aab}$ \\
\hline & Tronco'V' & 1,55 & $\mathrm{Aa}$ & 1,63 & $\mathrm{Aa}$ & 1,28 & $\mathrm{Ab}$ & 1,48 & $\mathrm{Aa}$ \\
\hline & Roçada & 1,42 & $A a b$ & 1,44 & $\mathrm{Ba}$ & 1,26 & $\mathrm{Ab}$ & 1,56 & $\mathrm{Aa}$ \\
\hline & Rolo-faca & 1,58 & $\mathrm{Aa}$ & 1,66 & $\mathrm{Aa}$ & 1,17 & $\mathrm{Ab}$ & 1,49 & $\mathrm{Aa}$ \\
\hline \multirow{4}{*}{$\begin{array}{c}\text { Microporos } \\
(\%)\end{array}$} & Grade & 17,85 & $\mathrm{Aa}$ & 17,77 & $\mathrm{Aa}$ & 15,52 & $\mathrm{Aa}$ & 13,64 & $\mathrm{Ba}$ \\
\hline & Tronco'V' & 18,55 & $\mathrm{Aa}$ & 16,23 & $\mathrm{Aa}$ & 12,16 & $\mathrm{Ab}$ & 18,87 & $\mathrm{Aa}$ \\
\hline & Roçada & 15,52 & $\mathrm{Aa}$ & 17,97 & $\mathrm{Aa}$ & 16,78 & $\mathrm{Aa}$ & 17,59 & $\mathrm{Aa}$ \\
\hline & Rolo-faca & 15,05 & $\mathrm{Ab}$ & 18,29 & $\mathrm{Aa}$ & 12,94 & $\mathrm{Ab}$ & 20,22 & $\mathrm{Aa}$ \\
\hline \multirow{4}{*}{$\begin{array}{c}\text { Macroporos } \\
(\%)\end{array}$} & Grade & 33,11 & $\mathrm{Ab}$ & 22,67 & Ad & 42,52 & $\mathrm{ABa}$ & 28,85 & Ac \\
\hline & Tronco' $\mathrm{V}$ ' & 29,31 & $\mathrm{Ab}$ & 23,23 & Ac & 46,50 & Aa & 23,11 & $\mathrm{Bc}$ \\
\hline & Roçada & 34,21 & $\mathrm{Aa}$ & 23,03 & $\mathrm{Ab}$ & 36,21 & $\mathrm{Ba}$ & 24,20 & $\mathrm{Bb}$ \\
\hline & Rolo-faca & 19,69 & $\mathrm{Bb}$ & 17,78 & $\mathrm{Bb}$ & 39,52 & $\mathrm{ABa}$ & 24,20 & $\mathrm{Bb}$ \\
\hline \multirow{4}{*}{$\begin{array}{c}\text { Porosidade } \\
\text { Total } \\
(\%)\end{array}$} & Grade & 50,96 & $A a b$ & 40,51 & $\mathrm{Ab}$ & 58,00 & $\mathrm{Aa}$ & 42,48 & $\mathrm{Ab}$ \\
\hline & Tronco' $V^{\prime}$ ' & 47,86 & $\mathrm{Ab}$ & 39,46 & Ac & 58,66 & $\mathrm{Aa}$ & 41,99 & Ac \\
\hline & Roçada & 49,72 & $\mathrm{Aa}$ & 41,00 & $\mathrm{Ab}$ & 53,00 & $\mathrm{Aa}$ & 41,79 & $\mathrm{Ab}$ \\
\hline & Rolo-faca & 34,74 & $\mathrm{Bb}$ & 36,06 & $\mathrm{Bb}$ & 52,46 & Aa & 44,43 & Aab \\
\hline
\end{tabular}

Médias seguidas com as mesmas letras minúsculas nas linhas e maiúsculas nas colunas não diferem significativamente entre si, pelo teste de Tukey, a 5\% de significância. 
TABELA 3 - Rendimento médio expresso em número de frutos, peso de frutos de citros, peso médio de frutos e produtividade estimada sob diferentes manejos da vegetação.

\begin{tabular}{ccccc}
\hline \multirow{2}{*}{ Tratamento } & \multicolumn{2}{c}{ Rendimento médio/planta $^{(1)}$} & $\begin{array}{c}\text { Peso médio } \\
\text { de frutos (kg) }\end{array}$ & $\begin{array}{c}\text { Produtividade }^{(2)} \\
\mathbf{t ~ h a}^{-1}\end{array}$ \\
\cline { 2 - 3 } $\mathbf{N}^{\mathbf{0}}$ de frutos & $\begin{array}{c}\text { Peso de } \\
\text { frutos }(\mathbf{k g})\end{array}$ & $39,08 \mathrm{a}$ & $0,104 \mathrm{~b}$ & $19,54 \mathrm{a}$ \\
(T1) Grade & $375 \mathrm{a}$ & $30,33 \mathrm{ab}$ & $0,112 \mathrm{ab}$ & $15,17 \mathrm{ab}$ \\
(T2) Tronco 'V' & $271 \mathrm{~b}$ & $28,58 \mathrm{~b}$ & $0,114 \mathrm{a}$ & $14,29 \mathrm{~b}$ \\
(T3) Roçada & $250 \mathrm{~b}$ & $36,58 \mathrm{ab}$ & $0,107 \mathrm{ab}$ & $18,29 \mathrm{ab}$ \\
\hline (T4) Rolo-faca & $343 \mathrm{ab}$ & 18,7 & 5,1 & 17,8 \\
\hline $\mathbf{C V \%}$ & 20,7 & &
\end{tabular}

Médias seguidas da mesma letra entre os tratamentos analisados não diferem significativamente entre si, pelo teste de Tukey, a 5\% de probabilidade. ${ }^{(1)}$ Média considerando valores obtidos de 12 plantas por tratamento. ${ }^{(2)}$ Estimativa considerando 500 plantas ha $^{-1}$, de acordo com espaçamento utilizado.

TABELA 4 - Valores de massa seca da parte aérea da vegetação espontânea, obtidos nos diferentes tratamentos, nas entrelinhas do pomar de citros.

\begin{tabular}{cc}
\hline Tratamento & Massa Seca $\left(\mathbf{M g ~ h a}^{-1}\right)$ \\
\hline T1 & $6,78 \mathrm{~A}$ \\
T2 & $7,82 \mathrm{~A}$ \\
T3 & $7,44 \mathrm{~A}$ \\
T4 & $5,70 \mathrm{~B}$ \\
\hline
\end{tabular}

Médias seguidas da mesma letra entre os tratamentos não diferem significativamente, pelo teste de Tukey, com nível de $5 \%$ de significância.

\section{CONCLUSÕES}

1-A fertilidade do solo teve aumento expressivo até a profundidade de $0,4 \mathrm{~m}$, devido à adubação com composto e biofertilizante no pomar orgânico de citros.

2-A resistência mecânica do solo à penetração e a densidade do solo são maiores no tráfego do rodado em comparação à projeção da copa, nas diferentes profundidades e tratamentos de manejo das plantas de cobertura do solo.

3-O tráfego de máquinas causa alterações nos atributos físicos do solo na região do rodado, entretanto não são impeditivos à produtividade de frutos das tangerineiras.

4-Devido à diminuição da competição por água pelas plantas espontâneas, o tratamento com gradagem apresenta produtividade de frutos superior ao tratamento com roçada.

\section{REFERÊNCIAS}

AULER, P.A.M.; FIDALSKI, J.; PAVAN, M.A.; NEVES, C.S.V.J. Produção de laranja 'Pera' em sistemas de preparo de solo e manejo nas entrelinhas. Revista Brasileira de Ciência do Solo, Viçosa, MG, v.32, n.1, p. 363-374, 2008.

AVILAN, L.; LEAL, F.; MENESES, L.; SUCRE, R.; GARCIA, M.L. Distribuición Del sistema radical de lãs cítricas em algunos suelos de Venezuela. Fruits D'Outre Mér, Paris, v.41, n.11, p. 655-668, 1980.

BONINE, D. P.; JOÃO, P.L. Estudo da cadeia produtiva de citros no Vale do Caí-RS. Informativo da EMATER/RS: Série Realidade Rural, Porto Alegre, v.29, p.74, 2002.

COMISSÃO DE QUÍMICA E FERTILIDADE DO SOLO. Manual de adubação e calagem para os Estados do Rio Grande do Sul e Santa Catarina. 10.ed. Porto Alegre, 2004. 394p. 
CRUZ, A.C.R.; LIBARDI, P.L.; CARVALHO, L.A.; ROCHA, G.C. Balanço de água no volume de solo explorado pelo sistema radicular de uma planta de citros. Revista Brasileira de Ciência do Solo, Viçosa, MG, v.29, n.1, p. 497-506, 2005.

DEMATTÊ, J.L.; VITTI, G.C. Alguns aspectos relacionados ao manejo de solos para citrus. In: SEMINÁRIO INTERNACIONAL DE CITROS, 2., 1992, Campinas. Anais... Campinas: Fundação Cargill, p.67-99, 1992.

FÁVERO, C.; JUCKSCH, I.; COSTA, L.M.; ALVARENGA, R.C.; NEVES, J.C.L. Crescimento e acúmulo de nutrientes por plantas espontâneas e por leguminosas utilizadas para adubação verde. Revista Brasileira de Ciência do Solo, Viçosa, MG, v.24, n.1, p.171-177, 2000 .

FIDALSKI, J.; TORMENA, C.A.; SACAPIM, C.A. Espacialização vertical e horizontal dos indicadores de qualidade para um Latossolo Vermelho cultivado com citros. Revista Brasileira de Ciência do Solo, Viçosa, MG, v.31, n.1, p.9-19, 2007.

HOMMA, S.K. Efeito do manejo alternativo sobre a descompatação do solo, fungos micorrízicos arbusculares nativos e produção em pomar convencional de targor Murcott. 2005. 101f. Dissertação (Mestrado) - Escola Superior de Agricultura "Luiz de Queiroz", Universidade de São Paulo, Piracicaba, 2005.

IBGE. Produção agrícola municipal: 2007. Disponível em: <http://www.ibge.org.br>. Acesso em: 24 jan. 2009.

MAGALHÃES, A.F.J. Calagem e adubação. In: MAGAlHÃES, A.F.J. (Ed.). Cultivo de citros. Cruz das Almas: Embrapa Mandioca e Fruticultura Tropical, 2004. cap.5, p.36-43.

MALAVOLTA, E. Fertilizantes e seu impacto ambiental: micronutrientes e metais pesados, mitos, mistificação e fatos. São Paulo: Produquímica Indústria e Comércio, 1994. 153p.

MINATEL, A.L.G.; ANDRIOLI, I.; CENTURION, J.F.; NATALE, W. Efeitos da subsolagem e da adubação verde nas propriedades físicas do solo em pomar de citros. Engenharia Agrícola, Jaboticabal, v.26, n.1, p.86-95, 2006.
NEVES, C.S.V.J.; DECHEN, A.R. Sistemas de manejo de solo em pomar de tangerina 'Ponkan' sobre limão 'Cravo' em Latossolo Roxo. Laranja, Cordeirópolis, v.22, n.1, p.167-184, 2001.

NOOLING, J.W. Citrus root growth and soil pest management practices. Gainesville: University of Florida, Entomology and Nematology Department, 1992. 37p

PANZENHAGEN, N. V.; KOLLER, O. C.; VAQUIL, P. D.; SOUZA, P. V. D. de.; DAL SOGLIO, K. Aspectos técnicos-ambientais da produção orgânica na região citrícola do Vale do Rio Caí-RS. Ciência Rural, Santa Maria, v.38, n.1, p. 90-95, 2008.

PERIN, A.; GUERRA, J.G.M.; TEIXEIRA, M.G. Efeito da morfologia radicular de leguminosas herbáceas perenes na umidade de um argissolo. Seropédica: Embrapa Agrobiologia, 2000. 8p. (Comunicado Técnico, 44).

SILVA, A.P.; IMHOFF, S.; KAY, B. Plant response to mechanical resistance and air filled-porosity of soils under conventional and no-tillage system. Scientia Agrícola, Piracicaba, v.61, n.4, p.451-456, 2004.

SILVA, S.S.; CAMARGO, F.A.O.; CERETTA, C.A. Composição da fase sólida orgânica do solo. In: MEURER. E.J. (Ed). Fundamentos de química do solo. Porto Alegre: Genesis, p.73-98, 2004.

SIX, J.; FELLER, C.; DENEF, K.; OGLE, S.M.; SA, J.C. de M.; ALBRECHT, A. Soil organic matter, biota and aggregation in temperate and tropical soils: effects of no tillage. Agronomie, Paris, v. 22, p.755-775, 2002.

SOANE, B. D. The role of organic matter in soil compactibility: a review of some practical aspects. Soil and Tillage Research, Amsterdam, v.16, n.1-2, p.179- 201, 1990.

TERSI, F.E.A.; ROSA, S.M. A subsolagem no manejo de solo para os pomares de citros. Laranja, Cordeirópolis, v.16, n.2, p.289-298, 1995.

TREIN, C. R. The mechanics of soil compaction under wheels. 1995. $132 \mathrm{f}$. Tese (Doutorado) - Cranfield University, Silsoe, 1995.

WRIGHT, G.C.; McCLOSKEY, W.B.; TAYLOR, C. Managing orchard floor vegetation in flood-irrigated citrus groves. HortTechnology, Alexandria, v.13, n.4, 2003. Disponível em: <http://www.ashs.org/ horttech/>. Acesso em: 08 set. 2009. 